\title{
The Optimal Taxi Fleet Size Structure under Various Market Regimes When Charging Taxis with Link-Based Toll
}

\author{
Jincheng Zhu, ${ }^{1}$ Bin Shuai, ${ }^{1}$ Zhengfeng Huang, ${ }^{2}$ and Chaoyuan Sun ${ }^{3}$ \\ ${ }^{1}$ School of Transportation and Logistics, Southwest Jiaotong University, Chengdu 610031, China \\ ${ }^{2}$ School of Transportation, Southeast University, Nanjing 210096, China \\ ${ }^{3}$ School of Management and Economics, University of Electronic Science and Technology of China, Chengdu 610054, China
}

Correspondence should be addressed to Bin Shuai; shuaibin@home.swjtu.edu.cn

Received 27 August 2013; Accepted 25 October 2013

Academic Editor: Guiomar Martín-Herrán

Copyright (C) 2013 Jincheng Zhu et al. This is an open access article distributed under the Creative Commons Attribution License, which permits unrestricted use, distribution, and reproduction in any medium, provided the original work is properly cited.

\begin{abstract}
This paper investigates the optimal taxi fleet size structure under monopoly and oligopoly market regimes when taxis are charged with the link-based toll. We proposed a bilevel programming model to take account of the interaction between taxi fleet size and different traffic modes in the network. The upper level is to determine the optimal taxi fleet structure so as to maximize the profit of each taxi firm. The lower-level is a combined network equilibrium model (CNEM) representing the travelers' response to the equilibrium taxi fleet size structure when congestion toll is imposed on taxis. We show that the lower level problem can be formulated as an equivalent variational inequality formulation, which considers the hierarchical logit-based mode split, route choice, elastic demand, and vacant taxi distributions. The bilevel problem can be solved by an iterative heuristic solution algorithm, whereas the lower level model is solved by the block Gauss-Seidel decomposition approach together with method of successive averages. An application with numerical examples is presented to illustrate the effectiveness of the proposed model and algorithm, and some interesting findings are also provided.
\end{abstract}

\section{Introduction}

Congestion pricing has been widely accepted as an efficient traffic management policy to alleviate traffic congestion and has been implemented in many cities, for example, Singapore, London, and Stockholm since Pigou [1] first proposed the concept. Recently, an important issue in this field that whether taxis should be charged in the pricing zone has been attracting more and more attention in view of the high demand for taxis in most large cities and of its consistent impact on the traffic congestion. For instance, King and Peters [2] examined the impact of road pricing on both route choice and travel time of taxi trips between lower Manhattan and LaGuardia airport in Queens in the U.S.A The results indicated that, for passengers, usage of toll road gives rise to a cost greater than the benefit, unless one has extremely high value of time (about $\$ 170$ per hour). Nevertheless, in many cities such as Singapore and Hong Kong, aside from private cars, taxis also have to pay the charge when using toll road, because taxis make significant contribution to the traffic congestion and the external cost of taxis can not be internalized without toll. There is, however, a nonnegligible issue when charging taxis with toll, that is, the decline of profits for taxi firms. Due to the toll, the demand of taxi customers decreases (diverting to the public transit or private car), which leads to the drop in the profits of taxi firms. Thus, the firms would like to optimize the taxi fleet size or fare to maximize their profits.

Lots of studies have focused on the optimal taxi fleet size and fare structure for different objectives. Cairns and Liston-Heyes [3] examined the economic consequences of taxi regulations from the perspective of monopoly, social optimum, and second best scenarios, in which the taxi price, fleet size, and taxi working hour are the endogenous variables. Since Cairns and Liston-Heyes's work [3] is on the basis of a simple and abstract demand-supply model, ample attention has been paid to equilibrium modeling urban taxi services with a detailed spatial structure of taxi market [4-8]. 
Yang et al. [9] investigated the impacts of various types of taxi regulation on the market equilibrium when taxi fare and fleet size varied in the monopoly and competitive markets. Yang et al. [10] then further extended this work to incorporate the congestion externalities which are caused by the occupied and vacant taxi as well as normal vehicular traffic.

These studies, however, considered only the interaction between taxi service level (measured by taxi fleet size and fare) and taxi customer demand. In fact, the supply of taxi services and demand for normal traffic including private car and public transit are also interrelated. It is commonly acknowledged that if the number of private cars is large, fewer travelers would choose taxi because of the congested traffic and thus taxi firms tend to operate in a small fleet size. Inversely, as the taxi fleet size increases the road becomes more congested, and hence part of demand for private car shifts to taxi and public transit. Therefore, the intervening relationship between taxi fleet size and normal traffic should be taken into account.

In addition to the choice game between taxi and normal traffic, travelers' preference of taxi firm is also a key concern. In many cities, taxi services are provided by several independent taxi firms and different firms may provide varied taxi types; that is, there are luxury taxi and normal taxi which are allowed to charge at different levels. Even if the various taxi firms serve customers with the same taxi type, the customers also prefer to choose the taxi for which the customer-waiting time is minimal (i.e., the first available taxi). Therefore, the taxi market is an oligopoly in which the competition among taxi firms can be described as an $n$-player, noncooperative game.

Because the taxi service fare is generally set by the government in most cities, this paper concentrates on the optimal fleet size structure to maximize the profit of each firm in the monopoly and oligopoly markets when taxis are charged with toll. A bilevel programming model is proposed, in which the lower-level problem is formulated as the combined network equilibrium model (CNEM) considering the intervening relationship between taxi fleet size and different traffic modes including taxi, private car, and public transit. The lowerlevel model can thus represent the travelers' response to the equilibrium taxi fleet size structure. Since the interactions of network flows are asymmetric, we formulate the lowerlevel problem as an equivalent variational inequality that involves hierarchical logit-based mode split, route choice, elastic demand, and vacant taxi distributions. The upperlevel aims at profit maximization for each taxi firm. This study can thus offer some useful suggestions to the taxi firms and regulator when congestion toll is imposed on taxis.

The paper is structured as follows. Section 2 proposes the combined network equilibrium model (CNEM). Section 3 constructs an equivalent variatinal inequality for CNEM. Section 4 addresses the optimal taxi fleet size of each taxi firm in monopoly and oligopoly markets, respectively. Section 5 develops an iterative heuristic solution algorithm to solve the bi-level programming problem, while the lower-level formulation is solved by the block Gauss-Seidel decomposition approach together with method of successive averages. Section 6 provides a numerical example to demonstrate the effectiveness of the proposed methodology. Conclusions and future studies are given in Section 7.

\section{The Combined Network Equilibrium Model}

2.1. Preliminaries. Consider a road network $G(V, A)$, where $V$ is the set of nodes and $A$ is the set of links. $\bar{A}$ is the set of toll links, $\bar{A} \subseteq A$. Let $R$ and $S$ be the sets of origin and destination nodes and $I$ the set of taxi firms. Also, let the following superscripts " $p$," " $b$," " $o$," and " $v$ " indicate private car, bus, occupied taxi, and vacant taxi, respectively. In addition, we use $m \in M=(p, b,(o, i))$ to denote the combination of private car, bus, and occupied taxi of different taxi firms $i \in I$.

Let $q_{r s}$ be the total demand between OD pair $r \in R$ and $s \in S$, and then we have the following equation:

$$
q_{r s}=q_{r s}^{p}+q_{r s}^{b}+q_{r s}^{o}, \quad r \in R, s \in S,
$$

where $q_{r s}^{p}, q_{r s}^{b}$, and $q_{r s}^{o}$ represent the demands of private car, bus, and occupied taxi from origin $r \in R$ to destination $s \in S$, respectively. Furthermore, we provide the following demand conservation for occupied taxis:

$$
q_{r s}^{o}=\sum_{i \in I} q_{r s}^{o, i}, \quad r \in R, s \in S
$$

where $q_{r s}^{o, i}$ is the demand for taxi of firm $i \in I$ between origin zone $r \in R$ and destination zone $s \in S$.

And for each taxi firm, the following equations about origin and destination trips can be obtained:

$$
\begin{aligned}
& O_{r}^{o, i}=\sum_{s \in S} q_{r s}^{o, i}, \quad r \in R, \quad i \in I, \\
& D_{s}^{o, i}=\sum_{r \in R} q_{r s}^{o, i}, \quad s \in S, \quad i \in I,
\end{aligned}
$$

where $O_{r}^{o, i}$ and $D_{s}^{o, i}$ are demands for the taxi of firm $i \in I$ from origin zone $r \in R$ to destination zone $s \in S$, respectively.

\subsection{Generalized Costs}

2.2.1. Generalized Costs of the Private Car and Taxi Mode. Let $c_{a}^{p}, c_{a}^{o, i}$, and $c_{a}^{v, i}$ be the generalized costs on link $a \in A$ for private car, occupied taxi, and vacant taxi operated by firm $i \in I$, respectively. And all of them are supposed to be a linear function of link travel time $t_{a}$, link length $d_{a}$, and toll $y_{a}$. Let $b^{p}$ and $b^{v, i}$ be the operating costs per unit distance for private car and taxi of firm $i \in I$. Additionally, we use $b_{0}^{o, i}, b_{1}^{o, i}$, and $b_{2}^{o, i}$ to indicate the preliminary flag-fall charge per ride, mileagebased and delay-based fare that are charged to customers who take a taxi of firm $i \in I$.

Then, we have the following link-based cost structures when taxis as well as private car are tolled in the road pricing zone $[10,11]$ :

$$
\begin{gathered}
c_{a}^{p}=\lambda t_{a}\left(x_{a}\right)+b^{p} d_{a}+y_{a}, \quad a \in A, \\
c_{a}^{o, i}=\lambda t_{a}\left(x_{a}\right)+b_{1}^{o, i} d_{a}+b_{2}^{o, i}\left(t_{a}\left(x_{a}\right)-t_{a}^{0}\right)+y_{a}, \quad a \in A, \\
c_{a}^{v, i}=\lambda_{v} t_{a}\left(x_{a}\right)+b^{v, i} d_{a}+y_{a}, \quad a \in A,
\end{gathered}
$$


where $\lambda$ is the value of time (VOT) for users taking private car or taxi while $\lambda_{v}$ is VOT for taxi drivers. $t_{a}\left(x_{a}\right)$ is the travel time which is an increasing function of total flow $x_{a}$ on link $a \in A$. $t_{a}^{0}$ is the free flow travel time.

We then can present the total generalized cost for private car and taxi on path $k \in K_{r s}$ between origin $r \in R$ and destination $s \in S$, as follows:

$$
\begin{gathered}
C_{r s, k}^{p}=\sum_{a \in A} c_{a}^{p} \delta_{a, k}^{r s}, \quad r \in R, s \in S, k \in K_{r s}, \\
C_{r s, k}^{o, i}=\sum_{a \in A} c_{a}^{o, i} \delta_{a, k}^{r s}+b_{0}^{o, i}+\lambda_{o w} W_{r}^{o, i}, \\
r \in R, s \in S, k \in K_{r s}, i \in I, \\
C_{s r, k}^{v, i}=\sum_{a \in A} c_{a}^{v, i} \delta_{a, k}^{s r}, \quad r \in R, s \in S, k \in K_{s r}, i \in I,
\end{gathered}
$$

where $W_{r}^{o, i}$ is an endogenous variable representing the customer waiting time for taxi of firm $i \in I$ at zone $r \in R$. $\lambda_{\text {ow }}$ is the value of customer waiting time. According to Wong et al. [8], we can specify the expected customer waiting time as a function of the cruising vacant taxi hours and the area of the zone:

$$
W_{r}^{o, i}=\eta \frac{Z_{r}}{N_{r}^{v, i} w_{r}^{v, i}}, \quad r \in R, i \in I,
$$

where $Z_{r}$ is the area of zone $r \in R$ and $\eta$ is a model parameter which is common to all zones. $w_{r}^{v, i}$ is the waiting/searching time of vacant taxi operated by firm $i \in I$ in zone $r \in R$. $N_{r}^{v, i}$ is the number of vacant taxi meeting customers in zone $r$ per hour. Note that, at equilibrium, we have $N_{r}^{v, i}=O_{r}^{o, i}$. Therefore, (6) can be represented as follows:

$$
W_{r}^{o, i}=\eta \frac{Z_{r}}{O_{r}^{o, i} w_{r}^{v, i}}, \quad r \in R, i \in I
$$

2.2.2. Generalized Cost of the Bus Mode. It is assumed that buses operate on the dedicated bus lanes, and there is no interaction between bus and other travel modes. Also the capacity of bus is supposed to be large enough to meet the passenger demands and in each OD pair there is one bus line [12]. Therefore, we have the following generalized costs of bus passengers between origin $r \in R$ and destination $s \in S$, which is denoted as $C_{r s}^{b}$ :

$$
C_{r s}^{b}=\lambda_{b} T_{r s}+\zeta G_{r s}\left(q_{r s}^{b}\right)+\lambda_{b w} W_{r s}^{b}+\tau, \quad r \in R, s \in S,
$$

where $T_{r s}$ is the bus travel time. Under previous assumptions as well as the fixed bus schedule, $T_{r s}$ is thus constant here. $\lambda_{b}$ is the value of time for bus passengers. $G_{r s}\left(q_{r s}^{b}\right)$ is the crowding discomfort experienced by bus passengers, which is an increasing function with respect to the number of travelers choosing bus. $\zeta$ is the unit cost of discomfort. $W_{r s}^{b}$ is the waiting time of bus passengers and can be specified as $W_{r s}^{b}=$ $\alpha / F_{r s}$, where $F_{r s}$ is bus frequency. Furthermore, the passenger arrival obeys the uniform random distribution in congested condition, thus the value of $\alpha$ is $0.5 . \lambda_{b w}$ is the waiting time value of bus passenger. $\tau$ is the bus fare.

Additionally, the total flow on each link $a \in A$ can be calculated via the following equation

$$
\begin{aligned}
x_{a}= & \sum_{r \in R, s \in S} \sum_{k \in K_{r s}} \sum_{i \in I}\left(f_{r s, k}^{p}+f_{r s, k}^{o, i}\right) \delta_{a k}^{r s} \\
& +\sum_{s \in S, r \in R} \sum_{k \in K_{s r}} \sum_{i \in I} f_{s r, k}^{v, i} \delta_{a k}^{s r}, \quad a \in A,
\end{aligned}
$$

where $f_{r s, k}^{p}$ and $f_{r s, k}^{o, i}$ are the flow on path $k \in K_{r s}$ for private car and occupied taxi of firm $i \in I$, respectively. $f_{s r, k}^{v, i}$ is the flow of vacant taxi on path $k \in K_{s r}$, where $K_{r s}$ and $K_{s r}$ are the sets of paths between zone $r \in R$ and zone $s \in S$. $\delta_{a k}^{r s}$ and $\delta_{a k}^{s r}$ are link-path indicator variables which are 1 if link $a$ lies on path $k$ and 0 otherwise.

2.3. Taxi Service Time Constraint. It is assumed that totally $N^{i}$ cruising taxis operate in the network for each taxi firm $i \in I$. And the total taxi service time consists of occupied time (denoted as $T_{O}$ ) and empty time (denoted as $T_{V}$ ) within one unit period $(1 \mathrm{~h})$ operations of taxis. In a stationary state, the total occupied time of taxis from each firm is equal to the taxihours that complete all $q_{r s}^{o, i}, r \in R, s \in S, i \in I$, trips and thus can be given by

$$
T_{O}=\sum_{r \in R} \sum_{s \in S} q_{r s}^{o, i} h_{r s}^{o, i}, \quad i \in I,
$$

where $h_{r s}^{o, i}$ is the average travel time from origin $r \in R$ to destination $s \in S$ by taking taxi mode $i \in I$ and can be represented as $h_{r s}^{o, i}=\left(\sum_{k \in K_{r s}}\left(f_{r s, k}^{o, i} \sum_{a \in A} t_{a} \delta_{a, k}^{r s}\right)\right) / \sum_{k \in K_{r s}} f_{r s, k}^{o, i}$ [11]. The total empty time of taxis is comprised of moving times from zone $s \in S$ to zone $r \in R$ and waiting/searching times in the zones. Thus, this time can be given by

$$
T_{V}=\sum_{s \in S} \sum_{r \in R} q_{s r}^{v, i}\left(h_{s r}^{v, i}+w_{r}^{v, i}\right), \quad i \in I,
$$

where $q_{s r}^{v, i}$ is the number of vacant taxis operated by taxi firm $i \in I$ to search for customers between zone $s \in S$ and zone $r \in R$.

Therefore, the following constraint must be satisfied in term of the $1 \mathrm{~h}$ period [4]:

$$
\sum_{r \in R} \sum_{s \in S} q_{r s}^{o, i} h_{r s}^{o, i}+\sum_{s \in S} \sum_{r \in R} q_{s r}^{v, i}\left(h_{s r}^{v, i}+w_{r}^{v, i}\right)=N^{i}, \quad i \in I,
$$

where $N^{i}$ is the taxi fleet size of taxi firm $i \in I$.

2.4. Traffic Assignment. We suppose that each path from different traffic modes including private car, bus passenger, occupied taxi, and vacant taxi can reach user equilibrium. 

fied:

At equilibrium, the following conditions should be satis-

$$
\begin{aligned}
& C_{r s, k}^{m}=u_{r s}^{m}, \quad \text { if } f_{r s, k}^{m}>0, r \in R, s \in S, k \in K_{r s}, m \in M, \\
& C_{r s, k}^{m} \geq u_{r s}^{m}, \quad \text { if } f_{r s, k}^{m}=0, r \in R, s \in S, k \in K_{r s}, m \in M, \\
& C_{s r, k}^{v, i}=u_{s r}^{v, i}, \quad \text { if } f_{s r, k}^{v, i}>0, r \in R, s \in S, k \in K_{s r}, i \in I, \\
& C_{s r, k}^{v, i} \geq u_{s r}^{v, i}, \quad \text { if } f_{s r, k}^{v, i}=0, r \in R, s \in S, k \in K_{s r}, i \in I,
\end{aligned}
$$

where $u_{r s}^{m}$ and $u_{s r}^{v, i}$ are the minimum generalized costs for mode $m \in M$ and vacant taxi of firm $i \in I$ between origin $r \in R$ and destination $s \in S$, respectively.

2.5. Hierarchical Logit Mode Split. We propose a hierarchical logit mode choice function where the upper choice level presents a mode split between taxi and nontaxi traffic (including private car and public transit), and the lower choice level aims to obtain the proportion of customers choosing taxi of firm $i \in I$.

We now first focus on the following logit-based mode choice function, which is able to give the proportion of trips taken by the private car, bus, and taxi between origin $r \in R$ and destination $s \in S$ at equilibrium.

For private car we have

$$
\begin{aligned}
& P_{r s}^{p}=\left(\exp \left[-\beta_{1}\left(u_{r s}^{p}-\varphi_{r s}^{p}\right)\right]\right) \\
& \times\left(\exp \left[-\beta_{1}\left(u_{r s}^{p}-\varphi_{r s}^{p}\right)\right]\right. \\
&+\exp \left[-\beta_{1}\left(u_{r s}^{b}-\varphi_{r s}^{b}\right)\right] \\
&\left.+\exp \left[-\beta_{1}\left(L_{r s}^{o}-\varphi_{r s}^{o}\right)\right]\right)^{-1}, \\
& \quad r \in R, s \in S,
\end{aligned}
$$

where $P_{r s}^{p}$ is the proportion of trips taken by the private car. $L_{r s}^{o}=-\left(1 / \beta_{2}\right) \ln \sum_{i \in I} \exp \left[-\beta_{2}\left(u_{r s}^{o, i}-\varphi_{r s}^{o, i}\right)\right], r \in R, s \in S$, is the log sum of disutility for travelers choosing taxi mode. $\beta_{1}$ and $\beta_{2}$ are the dispersion coefficients. $\varphi_{r s}^{p}$ represents the attraction of private car for travelers between origin $r \in R$ and destination $s \in S$. By symmetry, we can get the proportion of trips taken by bus and taxi.

Then we have the number of travelers who take private car as follows:

$$
\begin{aligned}
q_{r s}^{p}=\left(\exp \left[-\beta_{1}\left(u_{r s}^{p}-\varphi_{r s}^{p}\right)\right]\right) & \\
\times & \left(\exp \left[-\beta_{1}\left(u_{r s}^{p}-\varphi_{r s}^{p}\right)\right]\right. \\
& +\exp \left[-\beta_{1}\left(u_{r s}^{b}-\varphi_{r s}^{b}\right)\right] \\
& \left.+\exp \left[-\beta_{1}\left(L_{r s}^{o}-\varphi_{r s}^{o}\right)\right]\right)^{-1} q_{r s}, \\
& \quad r \in R, s \in S .
\end{aligned}
$$

As taxi has been chosen as the transportation mode, the following logit-based function is proposed to obtain the proportion of taxis in firm $i \in I$ selected by travelers:

$$
P_{r s}^{o, i}=\frac{\exp \left[-\beta_{2}\left(u_{r s}^{o, i}-\varphi_{r s}^{o, i}\right)\right]}{\sum_{i^{\prime} \in I} \exp \left[-\beta_{2}\left(u_{r s}^{o, i^{\prime}}-\varphi_{r s}^{o, i^{\prime}}\right)\right]}, \quad r \in R, s \in S, i \in I .
$$

Thus, the number of travelers choosing taxis of firm $i \in I$ can be specified as follows:

$$
q_{r s}^{o, i}=\frac{\exp \left[-\beta_{2}\left(u_{r s}^{o, i}-\varphi_{r s}^{o, i}\right)\right]}{\sum_{i^{\prime} \in I} \exp \left[-\beta_{2}\left(u_{r s}^{o, i^{\prime}}-\varphi_{r s}^{o, i^{\prime}}\right)\right]} q_{r s}^{o}, \quad r \in R, s \in S, i \in I,
$$

where $u_{r s}^{o, i}$ is the minimum generalized costs for travelers choosing taxis of firm $i \in I$ between origin $r \in R$ and destination $s \in S . \varphi_{r s}^{o, i}$ is the attraction of taxi operated by firm $i \in I$. Note that the dispersion coefficients $\beta_{1}$ and $\beta_{2}$ should satisfy the condition that $\beta_{2} \geq \beta_{1}$ to keep the consistency of the hierarchical logit function [13].

2.6. Vacant Taxi Distributions. In addition to private car and occupied taxi, there are considerable amount of vacant taxis searching for customers, which is closely associated with the fleet size. In this section we construct the following logit type model to describe the vacant taxi behaviors on the road network [4]. As in Yang and Wong's paper [4], we suppose that every taxi driver attempts to spend the minimal expected search time in meeting customer, and the expected search time is assumed to be a random variable which is identically distributed with a Gumbel density function:

$$
P_{r / s}^{v, i}=\frac{\exp \left[-\sigma\left(u_{s r}^{v, i}+\lambda_{v} w_{r}^{v, i}\right)\right]}{\sum_{r^{\prime} \in R} \exp \left[-\sigma\left(u_{s r^{\prime}}^{v, i}+\lambda_{v} w_{r^{\prime}}^{v, i}\right)\right]}, \quad s \in S, r \in R, i \in I,
$$

where $P_{r / s}^{v, i}$ is the probability that vacant taxis of firm $i \in I$ depart from zone $s \in S$ and meet the customer in zone $r \in R . \sigma$ is a nonnegative parameter reflecting the degree of uncertainty for taxi drivers on customer demand and on taxi services of the whole market.

Furthermore, in a steadystate of equilibrium, every customer is eventually able to take a taxi after waiting and searching, and all occupied taxis will become available when passengers arrive at destinations [8]. Thus, we have the following constraints:

$$
\begin{gathered}
\sum_{r \in R} q_{s r}^{v, i}=D_{s}^{o, i}, \quad s \in S, i \in I, \\
\sum_{s \in S} q_{s r}^{v, i}=\sum_{s \in S} D_{s}^{o, i} \cdot P_{r / s}^{v, i}=O_{r}^{o, i}, \quad r \in R, i \in I .
\end{gathered}
$$

2.7. Elastic Demand. Note that taxi fleet size has an impact on the total demands in view of the fact that as fleet size increases the total demands decrease due to the congested road. 
Thus a demand function is presented here to describe the elasticity of the OD demands:

$$
q_{r s}=D_{r s}\left(u_{r s}\right), \quad r \in R, s \in R,
$$

where $q_{r s}$ is the total demand between origin $r \in R$ and destination $s \in S$, which is supposed to be a continuously and strictly decreasing function of users' minimum perceived generalized costs $u_{r s}$ :

$$
\begin{gathered}
u_{r s}=-\frac{1}{\beta_{1}} \ln \left\{\exp \left[-\beta_{1}\left(u_{r s}^{p}-\varphi_{r s}^{p}\right)\right]+\exp \left[-\beta_{1}\left(u_{r s}^{b}-\varphi_{r s}^{b}\right)\right]\right. \\
\left.+\exp \left[-\beta_{1}\left(L_{r s}^{o}-\varphi_{r s}^{o}\right)\right]\right\} \quad r \in R, s \in R .
\end{gathered}
$$

Correspondingly, $u_{r s}=D_{r s}^{-1}\left(q_{r s}\right)$ is the inverse demand function.

\section{An Equivalent Variational Inequality Program}

Due to the asymmetric interactions of network flows caused by the delay-based taxi charge, a variational inequality (VI) program is used in this section which is equivalent to the aforementioned combined network equilibrium model. It is worth noting that the model proposed here is different from that in Wong et al. [11]. Our model incorporates the impact of endogenous variable, taxi fleet size on all the traffic modes and total demands. In particular, the variable demands are taken into account, because total demands would be affected as taxi fleet size varies. Furthermore, we also consider the effect of charging taxis with toll on the profits of taxi firms. In Wong et al's work [11], however, the taxi fleet size is given as a normal parameter with a given OD demand pattern and the congestion toll is not included as well.

The feasible region $\Omega$ of our VI formulation is stated as below and related dual variables are also provided in the brackets:

$$
\begin{gathered}
\sum_{s \in S} q_{s r}^{v, i}=O_{r}^{o, i}, \quad r \in R, i \in I, \quad\left(e_{r}^{i}\right), \\
\sum_{r \in R} q_{s r}^{v, i}=D_{s}^{o, i}, \quad s \in S, i \in I, \quad\left(a_{s}^{i}\right), \\
\sum_{i \in I} q_{r s}^{o, i}=q_{r s}^{o}, \quad r \in R, s \in S, \quad\left(L_{r s}^{o}\right), \\
q_{r s}=q_{r s}^{p}+q_{r s}^{b}+q_{r s}^{o}, \quad r \in R, s \in S, \quad\left(\gamma_{r s}\right), \\
\sum_{k \in K_{r s}} f_{r s, k}^{m}=q_{r s}^{m}, \quad r \in R, s \in S, m \in M, \quad\left(u_{r s}^{m}\right), \\
\sum_{k \in K_{s r}} f_{s r, k}^{v, i}=q_{s r}^{v, i}, \quad s \in S, r \in R, \quad\left(u_{s r}^{v, i}\right), \\
f_{r s, k}^{m} \geq 0, \quad r \in R, s \in S, m \in M, k \in K_{r s}, \quad\left(\chi_{r s, k}^{m}\right), \\
f_{s r, k}^{v, i} \geq 0, \quad r \in R, s \in S, i \in I, k \in K_{s r}, \quad\left(\chi_{s r, k}^{v, i}\right), \\
q_{r s}^{m} \geq 0, \quad q_{s r}^{v, i} \geq 0, r \in R, s \in S, m \in M, i \in I .
\end{gathered}
$$

Constraints (22) and (23) are the conservation conditions of flow for vacant taxis. Equation (24) is the conservation of flow for occupied taxis. Equation (25) is the conservation equation for total demand. Equations (26) and (27) show that the sum of all path flows for private car, bus, occupied taxi, and vacant taxi should be equal to their demands, respectively. Equations (28), (29), and (30) are the nonnegativity constraints on path flows and demands, respectively.

With the feasible region above, the VI problem can be stated as follows. Find $\left(f_{r s, k}^{m, *}, f_{s r, k}^{v, i^{*}}, q_{r s}^{*}, q_{r s}^{m, *}, q_{s r}^{v, i^{*}}\right) \in \Omega$, which satisfies

$$
\begin{aligned}
& \sum_{r \in R, s \in S} \sum_{m \in M} \sum_{k \in K_{r s}} C_{r s, k}^{m}\left(f^{*}\right)\left(f_{r s, k}^{m}-f_{r s, k}^{m, *}\right) \\
& +\sum_{s \in S, r \in R} \sum_{k \in K_{s r}} \sum_{i \in I} C_{s r, k}^{v, i}\left(f^{*}\right)\left(f_{s r, k}^{v, i}-f_{s r, k}^{v, i^{*}}\right) \\
& +\sum_{r \in R, s \in S} \sum_{\psi \in\{p, b, o\}}\left(\frac{1}{\beta_{1}} \ln \frac{q_{r s}^{\psi, *}}{q_{r s}}-\varphi_{r s}^{\psi}\right)\left(q_{r s}^{\psi}-q_{r s}^{\psi, *}\right) \\
& +\sum_{r \in R, s \in S} \sum_{i \in I}\left(\frac{1}{\beta_{2}} \ln \frac{q_{r s}^{o, i^{*}}}{q_{r s}^{o}}-\varphi_{r s}^{o, i}\right)\left(q_{r s}^{o, i}-q_{r s}^{o, i^{*}}\right) \\
& +\sum_{s \in S, r \in R} \sum_{i \in I} \frac{1}{\sigma} \ln q_{s r}^{v, i^{*}}\left(q_{s r}^{v, i}-q_{s r}^{v, i^{*}}\right) \\
& \quad-\sum_{r \in R, s \in S} D_{r s}^{-1}\left(q_{r s}^{*}\right)\left(q_{r s}-q_{r s}^{*}\right) \geq 0,
\end{aligned}
$$

where $C_{r s, k}^{m}$ and $C_{s r, k}^{v, i}, r \in R, s \in S, m \in M$, and $k \in K_{r s}$, are defined by (5).

Proposition 1. The optimality conditions of the proposed variational inequality (VI) program are equivalent to the combined network equilibrium model in Section 2.

Proof. The KKT conditions of the variational inequality formulation (31) are given below:

$$
\begin{gathered}
f_{r s, k}^{m}: C_{r s, k}^{m}-u_{r s}^{m}-\chi_{r s, k}^{m}=0 \\
r \in R, s \in S, m \in M, k \in K_{r s} \\
f_{s r, k}^{v, i}: C_{s r, k}^{v, i}-u_{s r}^{v, i}-\chi_{s r, k}^{v, i}=0, \quad r \in R, s \in S, k \in K_{s r}, i \in I, \\
q_{r s}^{p}: \frac{1}{\beta_{1}} \ln \frac{q_{r s}^{p}}{q_{r s}}-\varphi_{r s}^{p}-\gamma_{r s}+u_{r s}^{p}=0, \quad r \in R, s \in S, \\
q_{r s}^{b}: \frac{1}{\beta_{1}} \ln \frac{q_{r s}^{b}}{q_{r s}}-\varphi_{r s}^{b}-\gamma_{r s}+u_{r s}^{b}=0, \quad r \in R, s \in S, \\
q_{r s}^{o}: \frac{1}{\beta_{1}} \ln \frac{q_{r s}^{o}}{q_{r s}}-\varphi_{r s}^{o}-\gamma_{r s}+L_{r s}^{o}=0, \quad r \in R, s \in S
\end{gathered}
$$




$$
\begin{gathered}
q_{r s}^{o, i}: \frac{1}{\beta_{2}} \ln \frac{q_{r s}^{o, i}}{q_{r s}^{o}}-\varphi_{r s}^{o, i}+u_{r s}^{o, i}-L_{r s}^{o}=0, \quad r \in R, s \in S, \\
q_{s r}^{v, i}: \frac{1}{\sigma} \ln q_{s r}^{v, i}+e_{r}^{i}+a_{s}^{i}+u_{s r}^{v, i}=0, \quad s \in S, r \in R, \\
q_{r s}:-D_{r s}^{-1}\left(q_{r s}\right)+\gamma_{r s}=0, \quad r \in R, s \in S .
\end{gathered}
$$

The complementarity conditions are

$$
\begin{gathered}
f_{r s, k}^{m} \cdot \chi_{r s, k}^{m}=0, \quad r \in R, s \in S, m \in M, k \in K_{r s}, \\
f_{s r, k}^{v, i} \cdot \chi_{s r, k}^{v, i}=0, \quad r \in R, s \in S, k \in K_{s r}, i \in I, \\
\chi_{r s, k}^{m} \geq 0, \quad r \in R, s \in S, m \in M, k \in K_{r s}, \\
\chi_{s r, k}^{v, i} \geq 0, \quad r \in R, s \in S, k \in K_{s r}, i \in I .
\end{gathered}
$$

From (40) and (42), we have $\chi_{r s, k}^{m}=0$ if $f_{r s, k}^{m}>0$. Then, according to (32), we can obtain that $C_{r s, k}^{m}=u_{r s}^{m}$. And if $f_{r s, k}^{m}=$ $0, \chi_{r s, k}^{m} \geq 0$, then $C_{r s, k}^{m} \geq u_{r s}^{m}$. Therefore, we have the following conditions:

$$
\begin{aligned}
& C_{r s, k}^{m}=u_{r s}^{m}, \quad \text { if } f_{r s, k}^{m}>0, r \in R, s \in S, k \in K_{r s}, m \in M, \\
& C_{r s, k}^{m} \geq u_{r s}^{m}, \quad \text { if } f_{r s, k}^{m}=0, r \in R, s \in S, k \in K_{r s}, m \in M,
\end{aligned}
$$

which implies that the route choices of private car, bus commuters, and occupied taxis of each firm follow user equilibrium. Similarly, we can demonstrate that the route choice of vacant taxis also satisfies the user equilibrium condition by utilizing (41), (43), and (33).

From (37) we have

$$
\frac{q_{r s}^{o, i}}{q_{r s}^{o}}=\exp \left[\beta_{2}\left(\varphi_{r s}^{o, i}-u_{r s}^{o, i}+L_{r s}^{o}\right)\right], \quad r \in R, s \in S .
$$

Taking the sum of $i$ in both sides gives rise to

$$
\begin{array}{r}
L_{r s}^{o}=-\frac{1}{\beta_{2}} \ln \sum_{i \in I} \exp \left[-\beta_{2}\left(u_{r s}^{o, i}-\varphi_{r s}^{o, i}\right)\right], \\
r \in R, s \in S .
\end{array}
$$

Substituting (46) into (45) leads to

$$
\frac{q_{r s}^{o, i}}{q_{r s}^{o}}=\frac{\exp \left[-\beta_{2}\left(u_{r s}^{o, i}-\varphi_{r s}^{o, i}\right)\right]}{\sum_{i^{\prime} \in I} \exp \left[-\beta_{2}\left(u_{r s}^{o, i^{\prime}}-\varphi_{r s}^{o, i^{\prime}}\right)\right]}, \quad r \in R, s \in S, i \in I,
$$

which is the lower choice level of hierarchical logit mode split function, that is, (17).

From (34) we can see

$$
\frac{q_{r s}^{p}}{q_{r s}}=\exp \left[\beta_{1}\left(\varphi_{r s}^{p}+\gamma_{r s}-u_{r s}^{p}\right)\right], \quad r \in R, s \in S .
$$

By symmetry, we have the following equations according to (35) and (36):

$$
\begin{array}{ll}
\frac{q_{r s}^{b}}{q_{r s}}=\exp \left[\beta_{1}\left(\varphi_{r s}^{b}+\gamma_{r s}-u_{r s}^{b}\right)\right], & r \in R, s \in S, \\
\frac{q_{r s}^{o}}{q_{r s}}=\exp \left[\beta_{1}\left(\varphi_{r s}^{o}+\gamma_{r s}-L_{r s}^{o}\right)\right], & r \in R, s \in S .
\end{array}
$$

Equation (48) plus (49) plus (50) gives rise to

$$
\begin{array}{r}
\exp \left(\beta_{1} \gamma_{r s}\right)=(1)\left(\exp \left[-\beta_{1}\left(u_{r s}^{p}-\varphi_{r s}^{p}\right)\right]\right. \\
+\exp \left[-\beta_{1}\left(u_{r s}^{b}-\varphi_{r s}^{b}\right)\right] \\
\left.+\exp \left[-\beta_{1}\left(L_{r s}^{o}-\varphi_{r s}^{o}\right)\right]\right)^{-1}, \\
\quad r \in R, s \in S .
\end{array}
$$

Substituting (51) into (48) we can see

$$
\begin{aligned}
q_{r s}^{p}=\left(\exp \left[-\beta_{1}\left(u_{r s}^{p}-\varphi_{r s}^{p}\right)\right]\right) & \\
\times & \left(\exp \left[-\beta_{1}\left(u_{r s}^{p}-\varphi_{r s}^{p}\right)\right]\right. \\
& +\exp \left[-\beta_{1}\left(u_{r s}^{b}-\varphi_{r s}^{b}\right)\right] \\
& \left.+\exp \left[-\beta_{1}\left(L_{r s}^{o}-\varphi_{r s}^{o}\right)\right]\right)^{-1} q_{r s}, \\
& \quad r \in R, s \in S,
\end{aligned}
$$

which is the upper choice level of hierarchical logit mode split function, that is, (15).

From (51) we have

$$
\begin{aligned}
& \gamma_{r s}=-\frac{1}{\beta_{1}} \ln \left\{\exp \left[-\beta_{1}\left(u_{r s}^{p}-\varphi_{r s}^{p}\right)\right]\right. \\
&+\exp \left[-\beta_{1}\left(u_{r s}^{b}-\varphi_{r s}^{b}\right)\right] \\
&\left.+\exp \left[-\beta_{1}\left(L_{r s}^{o}-\varphi_{r s}^{o}\right)\right]\right\} \\
& r \in R, s \in S .
\end{aligned}
$$

Substituting (53) into (39) we have

$$
\begin{array}{r}
D_{r s}^{-1}\left(q_{r s}\right)=-\frac{1}{\beta_{1}} \ln \left\{\exp \left[-\beta_{1}\left(u_{r s}^{p}-\varphi_{r s}^{p}\right)\right]\right. \\
+\exp \left[-\beta_{1}\left(u_{r s}^{b}-\varphi_{r s}^{b}\right)\right] \\
\left.+\exp \left[-\beta_{1}\left(L_{r s}^{o}-\varphi_{r s}^{o}\right)\right]\right\}, \\
r \in R, s \in S,
\end{array}
$$

which indicates that the elastic demand function defined in Section 2.7 is satisfied.

Equation (38) can be rewritten as follows:

$$
q_{s r}^{v, i}=\exp \left[-\sigma\left(u_{s r}^{v, i}+a_{s}^{i}+e_{r}^{i}\right)\right], \quad r \in R, s \in S .
$$


Substituting (55) into (23) we have

$$
\exp \left(-\sigma a_{s}^{i}\right)=\frac{D_{s}^{o, i}}{\sum_{r \in R} \exp \left[-\sigma\left(u_{s r}^{v, i}+e_{r}^{i}\right)\right]}, \quad s \in S, i \in I .
$$

Then, substituting (56) into (55) we can see

$$
q_{s r}^{v, i}=\frac{\exp \left[-\sigma\left(u_{s r}^{v, i}+e_{r}^{i}\right)\right]}{\sum_{r^{\prime} \in R} \exp \left[-\sigma\left(u_{s r^{\prime}}^{v, i}+e_{r^{\prime}}^{i}\right)\right]} D_{s}^{o, i}, \quad r \in R, s \in S, i \in I .
$$

Comparing (57) with the logit-based vacant taxi distribution model equation (18), we know that $\lambda_{v} w_{r}^{v, i}$ is in connection with $e_{r}^{i}$. Similar to Wong et al. [8], we can calculate taxi waiting/searching time $w_{r}^{v, i}$ through (12), (22), (23), and (55) (for details, one can see Wong et al. [8]).

Since the constraints (22) to (30) are nonnegative and linear, and the continuous formulation (31) has the region of non-negative flows and OD demands, we can conclude that at least one solution to the VI program exists [11].

\section{Optimal Taxi Fleet Size Structure of Taxi Firms}

4.1. Profit Function of Taxi Firms. The taxi fleet size structure of taxi firms has significant effects on the behaviors of all the travelers including taxi customers and, thus, on the profits of taxi firms. The net profit of a taxi firm is the total revenue raised from taxi customer fares minus the total taxi operating costs. It is assumed that the cost of taxi operation for "occupied" service time is consistent with the "vacant" service time and $\xi^{i}$ is denoted as the operating cost per taxi per hour of service time for taxi firm $i \in I$. Consequently the total taxi operating cost of taxi firm $i \in I$ can be represented as $\xi^{i} N^{i}$ [9]. The net profit, $\Phi_{i}$, of taxi firm $i \in I$ then can be expressed as follows:

$$
\begin{aligned}
\Phi_{i}( & \left.\mathbf{N}, \mathbf{q}^{o, i}(\mathbf{N}), \mathbf{x}^{o, i}(\mathbf{N})\right) \\
= & \sum_{r \in R, s \in S} b_{0}^{o, i} q_{r s}^{o, i}(\mathbf{N})+\sum_{a \in A} b_{1}^{o, i} d_{a} x_{a}^{o, i}(\mathbf{N}) \\
& +\sum_{a \in A} b_{2}^{o, i}\left(t_{a}\left(x_{a}\right)-t_{a}^{0}\right) x_{a}^{o, i}(\mathbf{N})-\xi^{i} N^{i}, \quad i \in I,
\end{aligned}
$$

where $\mathbf{q}^{o, i}$ and $\mathbf{x}^{o, i}$ are the vectors representing the numbers of travelers choosing taxis of firm $i \in I$ and link flow for occupied taxis of firm $i \in I$, respectively.

4.2. Market Equilibrium. With the above-mentioned model and assumptions, we now investigate the optimal taxi fleet size structure under two scenarios: the monopoly market and oligopolistic competitive market.

4.2.1. Monopoly Market. The monopoly market here is defined as all the taxis in the network are operated by a single firm to which the monopoly rights to pick up customers in the market area are granted by the government, and the government also sets the taxi fare. Thus, under this monopoly system, the taxi firm would maximize its net profit generated from the fare charges. The upper-level program then aims to determine the optimal taxi fleet size so as to maximize the net profit:

$$
\max \Phi\left(N, \mathbf{q}^{o}(N), \mathbf{x}^{o}(N)\right)
$$

where $\mathbf{q}^{o}(N)$ and $\mathbf{x}^{o}(N)$ are obtained by solving the variational inequality program (31).

4.2.2. Oligopoly Market. It is commonly acknowledged that taxi services are generally provided by several independent operators in practice, which gives rise to a competition where each firm seeks to maximize its own profits. Because the taxi fare is set by regulator, each taxi firm would optimize its taxi fleet size. Then the Nash equilibrium among different taxi firms can be found by determining the equilibrium taxi fleet size structure such that the following inequalities are satisfied:

$$
\begin{array}{r}
\Phi_{i}\left(N^{i^{*}}, \mathbf{N}^{-i^{*}}, \mathbf{q}^{o, i}\left(N^{i^{*}}, \mathbf{N}^{-i^{*}}\right), \mathbf{x}^{o, i}\left(N^{i^{*}}, \mathbf{N}^{-i^{*}}\right)\right) \\
\geq \Phi_{i}\left(N^{i}, \mathbf{N}^{-i^{*}}, \mathbf{q}^{o, i}\left(N^{i}, \mathbf{N}^{-i^{*}}\right), \mathbf{x}^{o, i}\left(N^{i}, \mathbf{N}^{-i^{*}}\right)\right), \\
i \in \mathbf{I}, N^{i} \in \mathfrak{R}^{i} .
\end{array}
$$

Inequality (60) indicates that no one can increase its profit by unilaterally changing taxi fleet size under equilibrium situation. $N^{i^{*}}$ represents the optimal solution of taxi fleet size for firm $i$ and $\mathbf{N}^{-i^{*}}$ denotes the optimal solution of taxi fleet size for the other firms excluding $i . \mathfrak{R}^{i}$ is the strategy set of taxi firm i. $\mathbf{q}^{o}\left(N^{i^{*}}, \mathbf{N}^{-i^{*}}\right)$ and $\mathbf{x}^{o}\left(N^{i^{*}}, \mathbf{N}^{-i^{*}}\right)$ are given by solving variational inequality formulation (31).

For convenience, let $\mathbf{v}^{i}=\left(\mathbf{N}, \mathbf{q}^{o, i}, \mathbf{x}^{o, i}\right), Z^{i}\left(\mathbf{v}^{i}\right)=\nabla_{\mathbf{v}^{i}} \Phi_{i}\left(\mathbf{v}^{i}\right)$, $\mathbf{Z}(\mathbf{v})=\left(Z^{1}\left(\mathbf{v}^{1}\right), Z^{2}\left(\mathbf{v}^{2}\right), \ldots, Z^{I}\left(\mathbf{v}^{I}\right)\right), i \in I$. Then, the above Nash equilibrium problem (60) can be expressed as the following variational inequality program:

$$
\mathbf{Z}\left(\mathbf{v}^{*}\right)\left(\mathbf{v}-\mathbf{v}^{*}\right) \geq 0, \quad \mathbf{v} \in \Psi\left(\mathbf{v}^{*}\right)
$$

where $\Psi=\Pi_{i} \Psi^{i}$ and $\Psi^{i}$ is the strategy set of vector $\mathbf{v}^{i}$. Furthermore, the solution in variational inequality (61) also satisfies inequality (60) [14].

\section{Solution Algorithm}

An iterative heuristic solution algorithm is developed to solve the bi-level programming where the variational inequality programs (31) can be solved by the block Gauss-Seidel decomposition approach coupled with method of successive averages [15], whereas (61) is solved by the diagonalization method [16]. One can refer to Zhou et al. [17] for the details of the heuristic solution algorithm. The procedure of the algorithm is structured as below. 
Step 1. Let the iteration number $j=0$. Set an initial taxi fleet size structure $\mathbf{N}^{(j)}$.

Step 2. Compute auxiliary solution $\mathrm{U}^{(j)}$ by solving variational inequality problem (61) and (31).

Substep 1. Set $i=1$.

Substep 2. Compute the auxiliary solution $U^{i(j)}$ by solving lower-level problem (31) and the following linear program (62) which is equivalent to the variational inequality problem (61). Note that the genetic algorithm or simulated annealing algorithm can be used to solve this problem. And the lowerlevel formulation (31) is solved by the block Gauss-Seidel decomposition approach coupled with method of successive averages. It is also worth noting that, in each solution procedure, to get the next pattern of taxi fleet size for taxi firm $i$, we need to calculate the taxi waiting time, $w_{r}^{v, i}$, through (12), (22), (23), and (55) and get the demand $\mathbf{q}^{o, i}$ and link flow $\mathbf{x}^{o, i}$, which are then substituted into (62) to get the profit $\Phi_{i}$ :

$$
\begin{array}{r}
\max \Phi_{i}\left(U^{i(j)}, \mathbf{N}^{-i(j)}, \mathbf{q}^{o, i}\left(U^{i(j)}, \mathbf{N}^{-i(j)}\right), \mathbf{x}^{o, i}\left(U^{i(j)}, \mathbf{N}^{-i(j)}\right)\right), \\
i \in I .
\end{array}
$$

Substep 3. If $i<I$, then $i=i+1$ and return to Substep 2, otherwise go to Step 3.

Step 3. Utilize the method of successive averages to find the solution pattern of next iteration:

$$
\mathbf{N}^{(j+1)}=\mathbf{N}^{(j)}+\frac{1}{j+1}\left(\mathbf{U}^{(j)}-\mathbf{N}^{(j)}\right)
$$

Step 4. If $\left|N^{i(j+1)}-N^{i(j)}\right| \leq \varepsilon_{1}$ for all $i \in I$, where $\varepsilon_{1}$ is a preset tolerance, then stop. Otherwise let $j=j+1$ and return to Step 2.

Note that the method of successive averages is used in the proposed algorithm to get the solution pattern of next iteration, because many existing literatures, such as $[11,15]$, have shown that it can perform well even in a large-scale transport network and the stable solutions can always be obtained.

\section{Numerical Example}

In this section, we utilize a numerical example proposed by Zhu et al. [18] to illustrate the presented model and algorithm. The road network is depicted in Figure 1 with 4 OD pairs, 6 nodes, and 14 links. For the sake of simplicity, we suppose that there are two taxi firms in the oligopoly market let $i=1$ denote the taxi firm providing normal taxi service, and, $i=2$ represent the firm providing the luxury taxi service. Clearly, the fare for luxury taxi is higher than that for normal taxi. Also we let $i=1$ indicate the single taxi firm in the case of monopoly market. The bus lines are 1-2-4-6, 1-3-4, 5-6, and 5-3-4, respectively, which are represented as dotted lines in Figure 1.

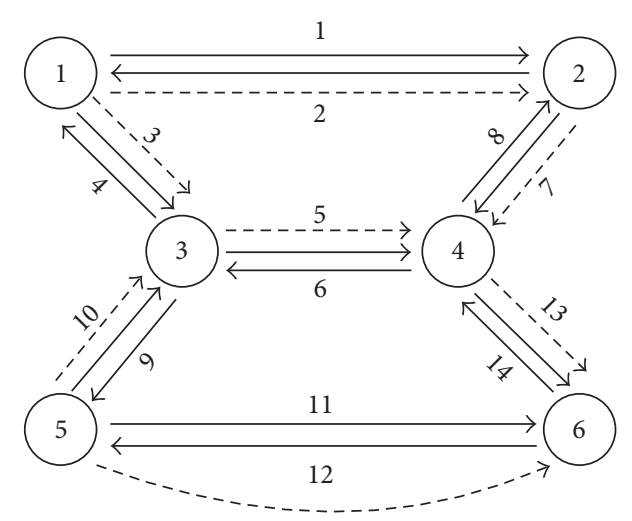

FIGURE 1: The network.

We assume the travel time function for each link follows the traditional BPR function:

$$
\begin{array}{r}
t_{a}\left(x_{a}\right)=t_{a}^{0}\left[1+0.15\left(\frac{x_{a}^{p}+\sum_{i \in I} x_{a}^{o, i}+\sum_{i \in I} x_{a}^{v, i}}{C_{a}}\right)^{4}\right], \\
a \in A,
\end{array}
$$

where the free flow travel time $t_{a}^{0}$ and link capacity $C_{a}$ as well as link length $d_{a}, a \in A$, are given in Table 1 .

The negative exponential demand function is

$$
q_{r s}=\bar{q}_{r s} \exp \left(-\kappa_{r s} u_{r s}\right), \quad r \in R, s \in S,
$$

where $\bar{q}_{r s}$ is the potential demand for each OD pair and $\kappa_{r s}$ is an elasticity parameter that represents the sensitivity of demand to generalized costs. Let $\bar{q}_{16}=4000 \mathrm{veh} / \mathrm{h}$, $\bar{q}_{14}=3000 \mathrm{veh} / \mathrm{h}, \bar{q}_{56}=4000 \mathrm{veh} / \mathrm{h}$, and $\bar{q}_{54}=3000 \mathrm{veh} / \mathrm{h}$, respectively. Also, let $\kappa_{16}=\kappa_{14}=\kappa_{56}=\kappa_{54}=0.03$. Similar to Huang [19], the following passenger crowding discomfort function is specified

$$
G_{r s}\left(q_{r s}^{b}\right)=\theta_{1}\left(q_{r s}^{b}\right)^{2}+\theta_{2} q_{r s}^{b}, \quad r \in R, s \in S,
$$

where $\theta_{1}$ and $\theta_{2}$ are positive parameters. In this numerical example, let $\theta_{1}$ and $\theta_{2}$ be 0.001 and 0.0001 , respectively. Furthermore, the bus frequency and bus travel time are shown in Table 2.

Other parameters are set as follows: $b^{p}=3 \$ / \mathrm{km} ; b_{0}^{o, 1}=$ $10 \$, b_{0}^{o, 2}=12 \$ ; b_{1}^{o, 1}=2 \$ / \mathrm{km}, b_{1}^{o, 2}=3 \$ / \mathrm{km} ; b_{2}^{o, 1}=30 \$ / \mathrm{h}$, $b_{2}^{o, 2}=35 \$ / \mathrm{h} ; b^{v, 1}=1.5 \$ / \mathrm{km}, b^{v, 2}=2 \$ / \mathrm{km} ; \lambda=60 \$ / \mathrm{h}, \lambda_{v}=$ $40 \$ / \mathrm{h}, \lambda_{o w}=120 \$ / \mathrm{h}, \lambda_{b}=30 \$ / \mathrm{h}, \lambda_{b w}=60 \$ / \mathrm{h} ; \tau=2 \$ ;$ $\xi_{1}=30 \$ / \mathrm{h}, \xi_{2}=35 \$ / \mathrm{h} ; \zeta=0.01 ; \varphi_{16}^{p}=10, \varphi_{16}^{b}=1, \varphi_{16}^{o}=8$, $\varphi_{14}^{p}=10, \varphi_{14}^{b}=1, \varphi_{14}^{o}=8, \varphi_{56}^{p}=10, \varphi_{56}^{b}=1, \varphi_{56}^{o}=8, \varphi_{54}^{p}=$ $10, \varphi_{54}^{b}=1, \varphi_{54}^{o}=8 ; \varphi_{16}^{o, 1}=2, \varphi_{16}^{o, 2}=5, \varphi_{14}^{o, 1}=2, \varphi_{14}^{o, 2}=5$, $\varphi_{56}^{o, 1}=2, \varphi_{56}^{o, 2}=5, \varphi_{54}^{o, 1}=2, \varphi_{54}^{o, 2}=5 ; \beta_{1}=0.06, \beta_{2}=0.06 ;$ $y=[7.8,1.2,4.4,5.6,7.7,4.5,7.8,1.4,7.8,7.8,7.8,1.3,7.7,7.8], \sigma=0.2$, $\eta Z_{r}=10, r \in R$.

We initially consider the monopoly scenario in which there is a single profit-maximizing firm operating the taxi services in the market. Figure 2 plots the contours of the taxi 
TABLE 1: Link free flow, capacity and length.

\begin{tabular}{lccccc}
\hline Link & $\begin{array}{c}\text { Star } \\
\text { node }\end{array}$ & $\begin{array}{c}\text { End } \\
\text { node }\end{array}$ & $t_{a}^{0}(\mathrm{~h})$ & $C_{a}(\mathrm{veh} / \mathrm{h})$ & $d_{a}(\mathrm{~km})$ \\
\hline 1 & 1 & 2 & 0.03 & 1200 & 2 \\
2 & 2 & 1 & 0.03 & 1200 & 2 \\
3 & 1 & 3 & 0.03 & 1200 & 2 \\
4 & 3 & 1 & 0.03 & 1200 & 2 \\
5 & 3 & 4 & 0.04 & 1200 & 3 \\
6 & 4 & 3 & 0.04 & 1000 & 3 \\
7 & 2 & 4 & 0.02 & 1800 & 1 \\
8 & 4 & 2 & 0.02 & 1800 & 1 \\
9 & 3 & 5 & 0.03 & 1200 & 2 \\
10 & 5 & 3 & 0.03 & 1200 & 2 \\
11 & 5 & 6 & 0.04 & 1200 & 3 \\
12 & 6 & 5 & 0.04 & 1200 & 3 \\
13 & 4 & 6 & 0.04 & 1200 & 3 \\
14 & 6 & 4 & 0.04 & 1200 & 3 \\
\hline
\end{tabular}

TABLE 2: Bus travel time and frequency.

\begin{tabular}{lccc}
\hline Origin & Destination & $\begin{array}{c}\text { Travel time } \\
T_{r s}(\mathrm{~h})\end{array}$ & $\begin{array}{c}\text { Bus frequency } \\
F_{r s}(\mathrm{veh} / \mathrm{h})\end{array}$ \\
\hline 1 & 6 & 0.3 & 10 \\
1 & 4 & 0.2 & 10 \\
5 & 6 & 0.15 & 10 \\
5 & 4 & 0.2 & 10 \\
\hline
\end{tabular}

profit when the taxi fleet size and fare varied. As we can see from the figure that three possible outcomes including positive profit, zero profit, and negative profit can be obtained with various combinations of fleet size and fare. Point $G$ in Figure 2 is the monopolistic solution where the optimal taxi fleet size is 803 veh, resulting in a profit of $\$ 14400$ per hour. It is also worth noting that as the fare increases the optimal fleet size decreases, which can be observed from the monopoly solution dashed curve in Figure 2. This can be explained by the fact that the customer demand decreases due to the higher taxi fare and thus the taxi firm will reduce the number of taxis. The maximum profit of $\$ 14641$ occurs at point $\mathrm{M}$, with optimal taxi fleet size of 780 veh and preliminary flag-fall charge per ride of $\$ 12$, respectively. Therefore, the moderate taxi fleet size and fare can improve the profit of taxi firm in the case of monopoly market.

We now look at the oligopolistic competitive solution which can be determined by solving (61) and (31) with the constraints (22) to (30). Figure 3 depicts the convergence of the proposed iterative heuristic algorithm for oligopoly market. We can see that the final convergent equilibrium point is achieved in approximately seven iterations for this numerical example.

Figure 4 shows the profit contours of the two taxi firms with taxi fleet size pattern. It can be observed in Figure 4 that point $\mathrm{H}$ is the oligopolistic solution with the optimal taxi fleet size of 657 for taxi firm 1 and 575 for taxi firm 2, giving rise

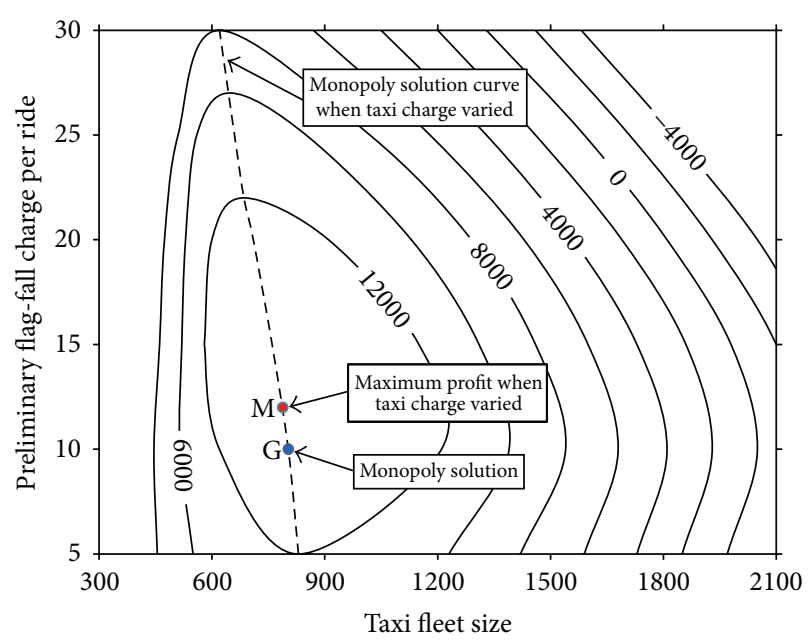

Figure 2: The profit of taxi firm for monopoly market as taxi fleet size and fare varied.

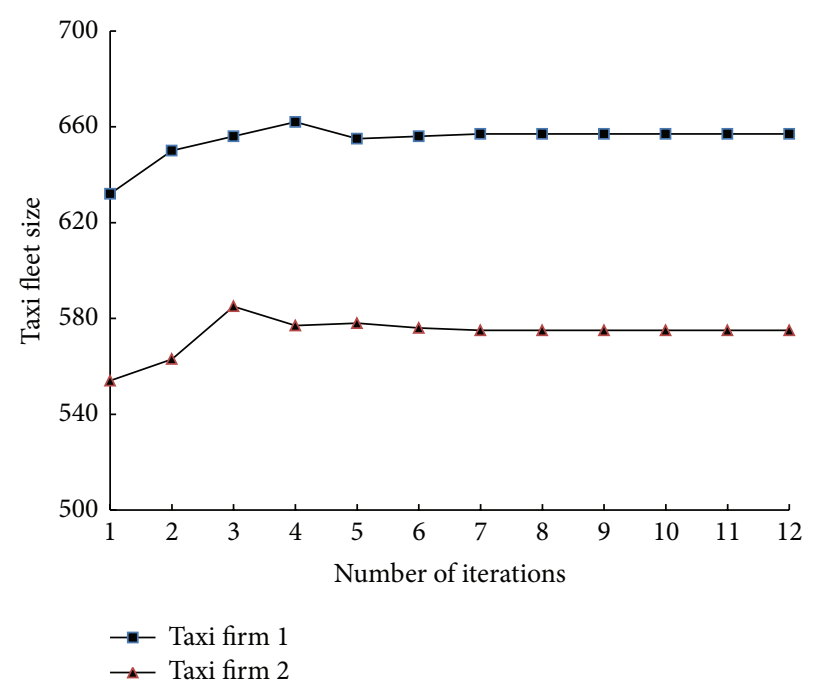

Figure 3: Convergence of the proposed algorithm.

to a profit of $\$ 7844$ per hour and $\$ 7886$ per hour for the two firms, respectively. It is noteworthy that the optimal taxi fleet size of taxi firm 1 is considerably larger than that of taxi firm 2. This is because as the two types of taxis are available more customers would like to choose the taxi type 1 because of the lower fare. Thus, taxi firm 1 tends to provide more taxis to meet the demands. The profit of taxi firm 2 is, however, as many as the profit of taxi firm 1 though the demand of taxi type 2 is obviously lower. This is because the higher taxi fare can make contribution to the profit.

Additionally, we also analyze the scenario when vacant taxis are exempted from tolling, as shown in Tables 3 and 4 . As we can see from the tables, the optimal taxi fleet size and profits of taxi firm in both monopoly market and oligopoly market increase, compared with the case that vacant taxis are charged with the toll. The reason is that as vacant taxis have to pay a congestion charge they would be less willing to cruise in the charging area or the area where toll is high. Thus, taxi 


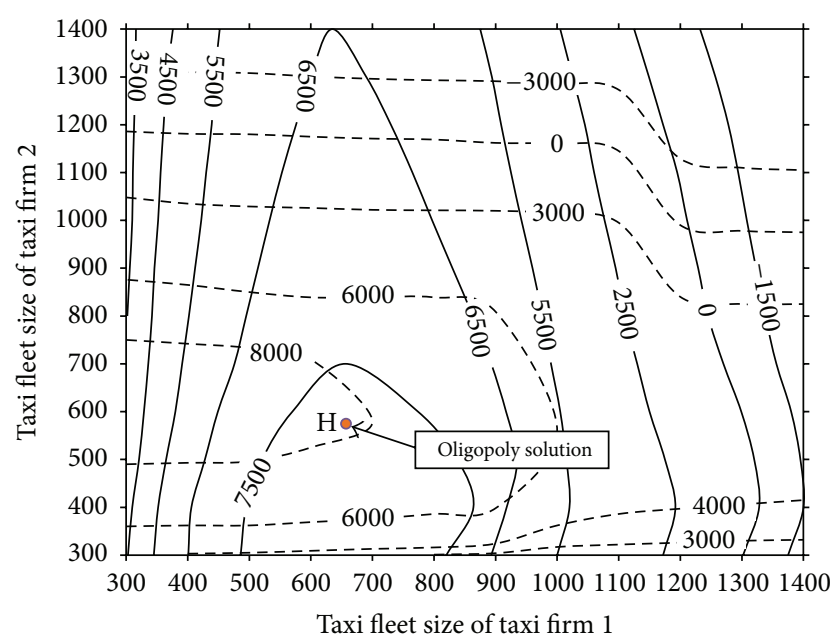

FIgURE 4: The profits of taxi firms for the oligopoly market as taxi fleet size varied.

TABLE 3: The monopolistic solution when vacant taxis are exempted from tolling.

\begin{tabular}{lc}
\hline \multicolumn{2}{c}{ Monopolistic solution } \\
\hline Optimal taxi fleet size & 910 veh \\
Taxi firm profit & $\$ 17350$ \\
\hline
\end{tabular}

TABLE 4: The oligopolistic solution when vacant taxis are exempted from tolling.

\begin{tabular}{lc}
\hline \multicolumn{2}{c}{ Oligopolistic solution } \\
\hline Optimal taxi fleet size of taxi firm 1 & 750 veh \\
Optimal taxi fleet size of taxi firm 2 & 645 veh \\
Profit of taxi firm 1 & $\$ 9238$ \\
Profit of taxi firm 2 & $\$ 9256$ \\
\hline
\end{tabular}

demand there decreases (diverting to private car and bus), leading to a smaller optimal taxi fleet size and lower profits of taxi firm.

\section{Conclusions}

A mathematical model is proposed to address the optimal taxi fleet size structure under different market regimes when taxis are charged with the link-based toll. The model is developed as a bi-level programming formulation that takes into account the intervening relationship between taxi fleet size and different traffic modes. The lower-level problem is a combined network equilibrium model formulated as an equivalent variational inequality describing the hierarchical logit-based mode split, route choice, elastic demand, and vacant taxi distributions. The upper level aims to determine the optimal taxi fleet size structure so as to maximize the profit of each firm. The bi-level problem is solved by a heuristic solution algorithm whereas the lower level can be solved by the block Gauss-Seidel decomposition approach together with method of successive averages.
The results of the numerical example show that the optimal taxi fleet size decreases with the fare, while the moderate fleet size and fare can maximize the profit of taxi firm in the case of monopoly market. For the oligopoly scenario, the demand for the normal taxis is considerably higher than that for the luxury taxis because of the lower fare. Nevertheless, the profits of these two firms are almost the same. Furthermore, the optimal taxi fleet size and profits of taxi firm in both monopoly market and oligopoly market would increase if vacant taxis are exempted from tolling.

This study offers some useful advice to the taxi firms and regulator when charging taxis with toll. Real-world transportation networks instead of the synthetic network will be tested in the future study.

\section{Acknowledgments}

The research is supported by the National Natural Science Foundation of China (no. 71173177). The authors would like to express their thanks to the anonymous reviewer, whose constructive suggestions and helpful comments have considerably improved the exposition of this paper.

\section{References}

[1] A. C. Pigou, Wealth and Welfare, Macmillan, London, UK, 1920.

[2] D. A. King and J. R. Peters, "Slow down, you move too fast: the use of tolls by taxicabs in New York city," in Proceedings of the 91st Annual Meeting of the Transportation Research Board, (Compendium of Papers, CD-ROM), 2012.

[3] R. D. Cairns and C. Liston-Heyes, "Competition and regulation in the taxi industry," Journal of Public Economics, vol. 59, no. 1, pp. 1-15, 1996.

[4] H. Yang and S. C. Wong, "A network model of urban taxi services," Transportation Research B, vol. 32, no. 4, pp. 235-246, 1998.

[5] S. C. Wong and H. Yang, "Network model of urban taxi services: improved algorithm," Transportation Research Record, no. 1623, pp. 27-30, 1998.

[6] H. Yang, Y. W. Lau, S. C. Wong, and H. K. Lo, "A macroscopic taxi model for passenger demand, taxi utilization and level of services," Transportation, vol. 27, no. 3, pp. 317-340, 2000.

[7] J. M. Xu, S. C. Wong, H. Yang, and C. O. Tong, "Modeling level of urban taxi services using neural network," Journal of Transportation Engineering, vol. 125, no. 3, pp. 216-223, 1999.

[8] K. I. Wong, S. C. Wong, and H. Yang, "Modeling urban taxi services in congested road networks with elastic demand," Transportation Research B, vol. 35, no. 9, pp. 819-842, 2001.

[9] H. Yang, S. C. Wong, and K. I. Wong, "Demand-supply equilibrium of taxi services in a network under competition and regulation," Transportation Research B, vol. 36, no. 9, pp. 799819, 2002.

[10] H. Yang, M. Ye, W. H. Tang, and S. C. Wong, "Regulating taxi services in the presence of congestion externality," Transportation Research A, vol. 39, no. 1, pp. 17-40, 2005.

[11] K. I. Wong, S. C. Wong, H. Yang, and J. H. Wu, "Modeling urban taxi services with multiple user classes and vehicle modes," Transportation Research B, vol. 42, no. 10, pp. 985-1007, 2008. 
[12] R. Shi and Z. Li, "Pricing of multimodal transportation networks under different market regimes," Journal of Transportation Systems Engineering and Information Technology, vol. 10, no. 5, pp. 91-97, 2010.

[13] J. D. Ortuzar and L. G. Willumsen, Modeling Transport, John Wiley \& Sons, New York, NY, USA, 2nd edition, 1996.

[14] E. Cavazzuti, M. Pappalardo, and M. Passacantando, "Nash equilibria, variational inequalities, and dynamical systems," Journal of Optimization Theory and Applications, vol. 114, no. 3, pp. 491-506, 2002.

[15] M. Florian, J. H. Wu, and S. He, "A multi-class multi-mode variable demand network equilibrium model with hierarchical logit structures," in Transportation and Network Analysis: Current Trends-Miscellanea in Honor of Michael Florian, P. Marcotte and M. Gendreau, Eds., vol. 63 of Applied Optimization, pp. 119133, Kluwer Academic, London, UK, 2002.

[16] P. T. Harker, "A variational inequality approach for the determination of oligopolistic market equilibrium," Mathematical Programming, vol. 30, no. 1, pp. 105-111, 1984.

[17] J. Zhou, W. H. K. Lam, and B. G. Heydecker, "The generalized Nash equilibrium model for oligopolistic transit market with elastic demand," Transportation Research B, vol. 39, no. 6, pp. 519-544, 2005.

[18] J. C. Zhu, F. Xiao, and X. B. Liu, “Taxis in road pricing zone: should they pay the congestion charge?" submitted to Journal of Advanced Transportation.

[19] H. J. Huang, "Pricing and logit-based mode choice models of a transit and highway system with elastic demand," European Journal of Operational Research, vol. 140, no. 3, pp. 562-570, 2002. 


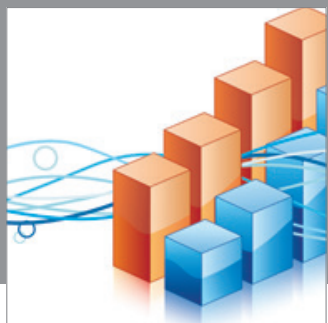

Advances in

Operations Research

mansans

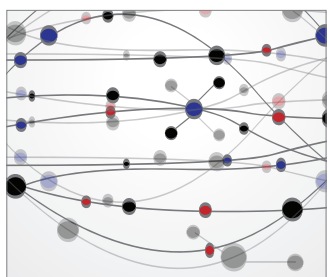

The Scientific World Journal
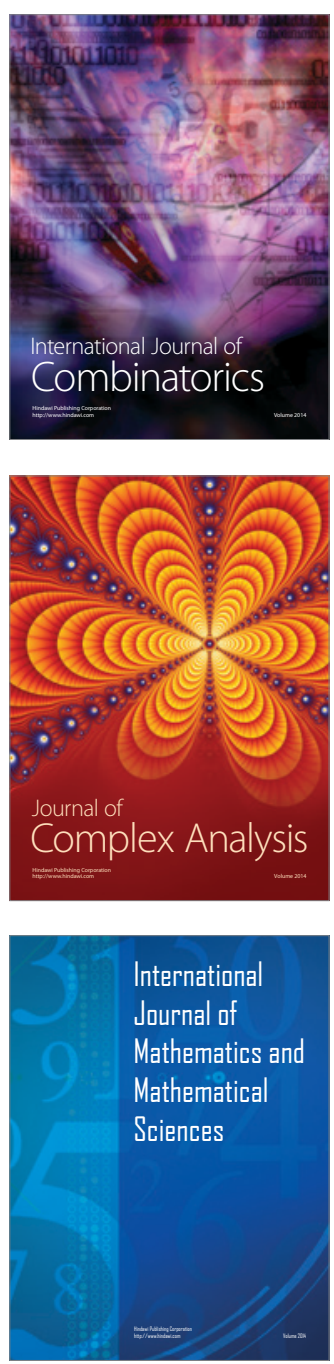
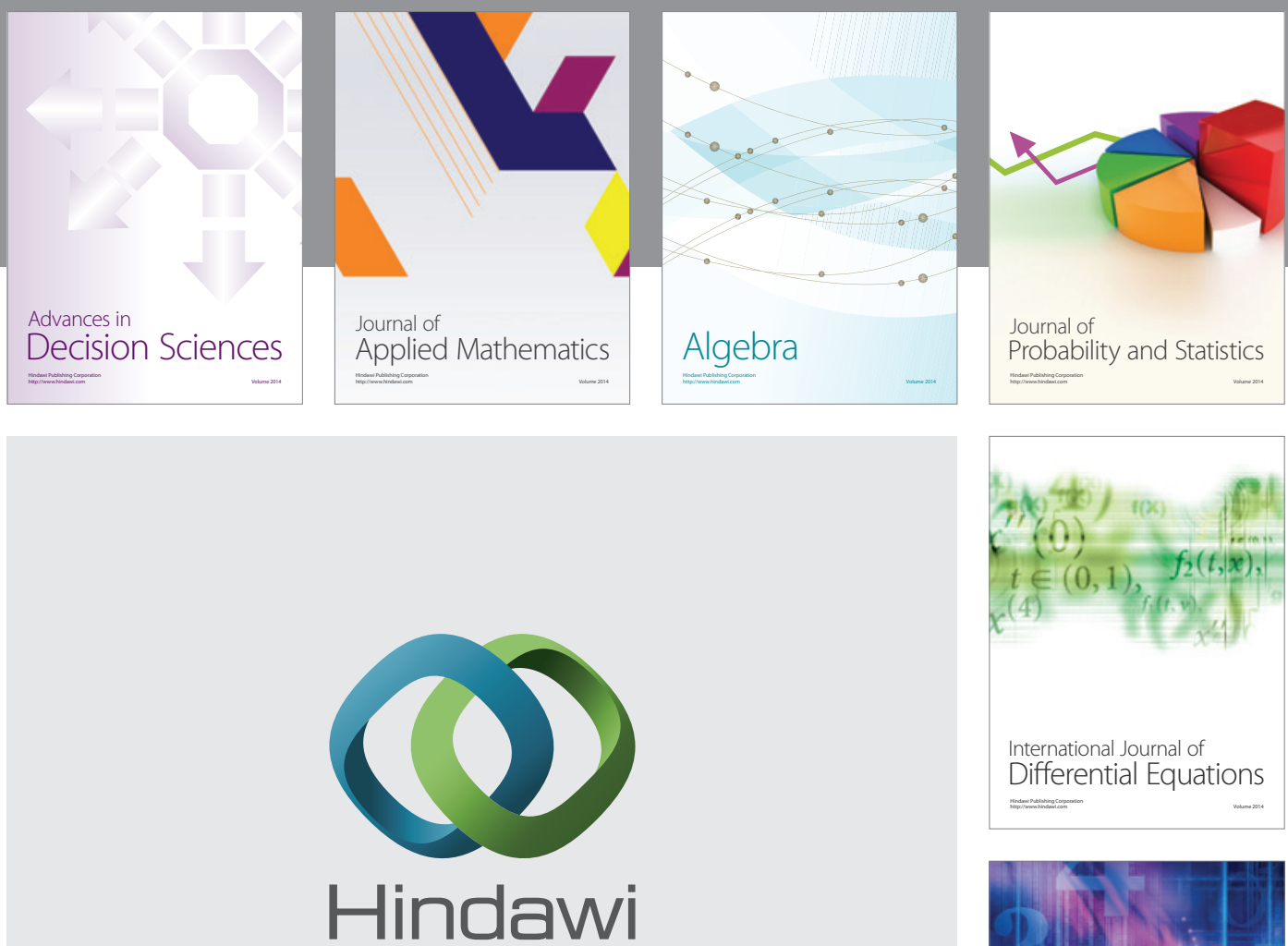

Submit your manuscripts at http://www.hindawi.com
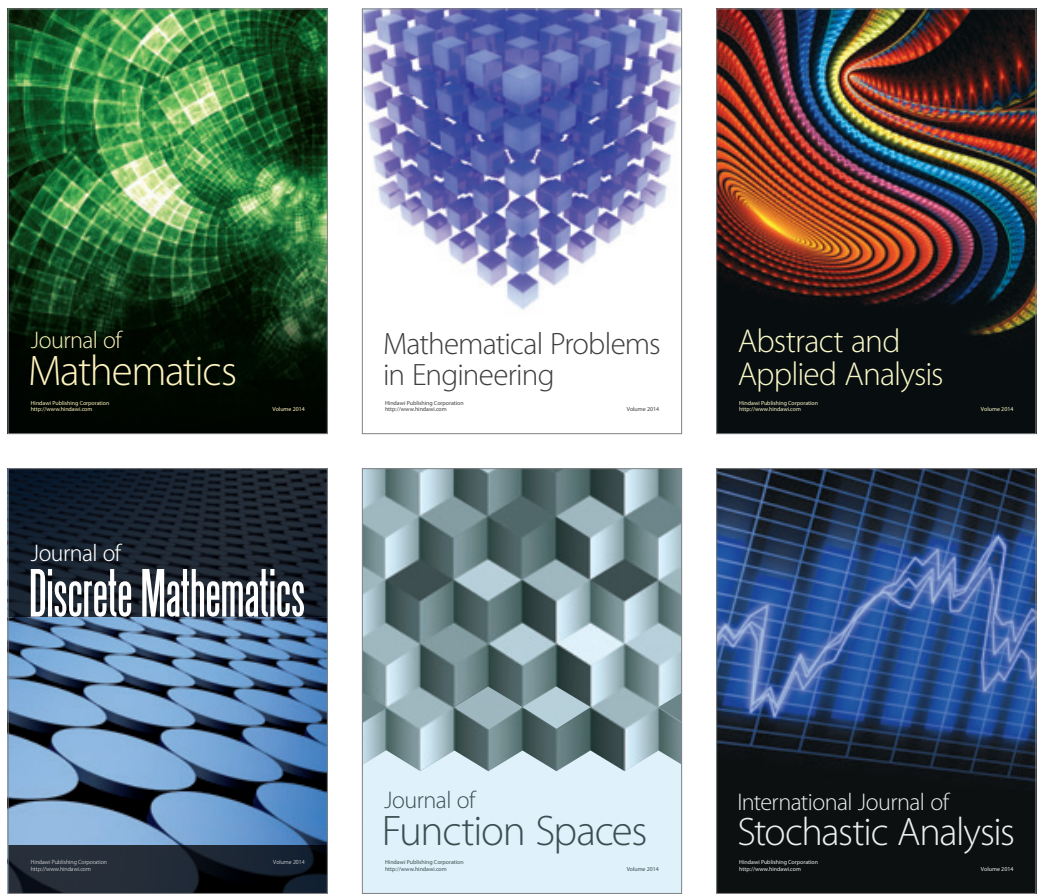

Journal of

Function Spaces

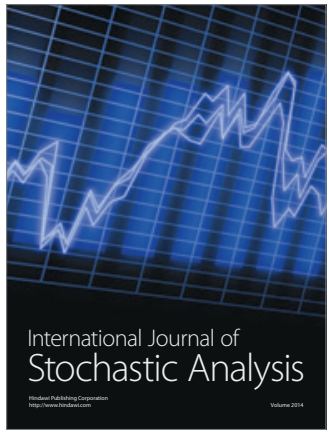

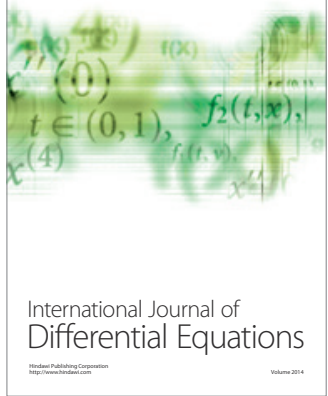
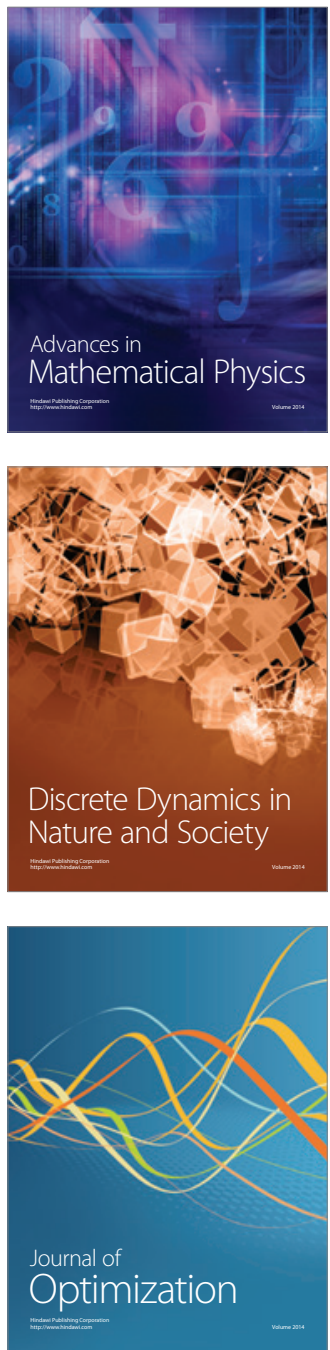